\title{
THE EFFECT OF HEMORRHAGE AND OF OCCLUSION OF THE CAROTID ARTERIES ON VASOMOTOR IRRITABILITY *
}

\author{
R. G. HOSKINS, Ph.D. \\ Associate Professor of Physiology, Northwestern University Medical School \\ WALTER N. ROWLEY AND CURTICE ROSSER \\ CHICAGO
}

The mechanism which controls blood pressure is one of the most complicated with which the physician has to deal. Arterial pressure depends on two factors-the amount of excitation and the irritability and the resistance to flow in the blood vessels. Peripheral resistance in turn depends to some extent on the elasticity of the arterial walls and the relative viscosity of the blood, but more particularly on the aggregate bore of the terminal arterial channels-the arterioles. This bore is quite variable and depends on several factors. The chemical composition of the blood itself has an important influence on the muscle cells of the vessels. The presence or absence of epinephrin is an example in point. The concentration of carbon dioxid is another factor the importance of which has only lately begun to be realized. The temperature of the blood is probably also of some practical significance. But the most important influence is that exerted by the vasomotor nervous system on the muscle cells of the arterioles. This system is essentially an intricate complex of reflex arcs. The term vasomotor apparatus is used in this paper to include both the nervous structures proper and the muscle cells to which the nerve impulses are finally delivered.

The activity of the vasomotor apparatus at any given moment depends on two factors-the amount of excitation and the irritability of the various parts of the apparatus. For example, blood pressure may be lowered either by putting the subject at rest, i. e., lessening the excitation, or by narcotizing some essential part of the apparatus. Although a great amount of work has been done on the blood pressure as an entity, few attempts have been made to study specifically the influence of different conditions on vasomotor irritability. It is altogether likely that a clean-cut knowledge of this matter would aid materially in giving us an understanding of the essential nature of

* Submitted for publication, May 10, 1915.

* From the Laboratory of Physiology of the Northwestern University Medical School, Chicago. 
surgical shock and sleep as well as various clinical cases of hypertension and hypotension.

For some time past the efforts of our laboratory have been directed largely to studies along this line. Partictilarly, the relation of various ductless glands to vasomotor irritability ${ }^{1}$ has been investigated. This paper, which is a continuation of the series, is concerned with the influence of certain circulatory conditions themselves on the vasomotor reactions.

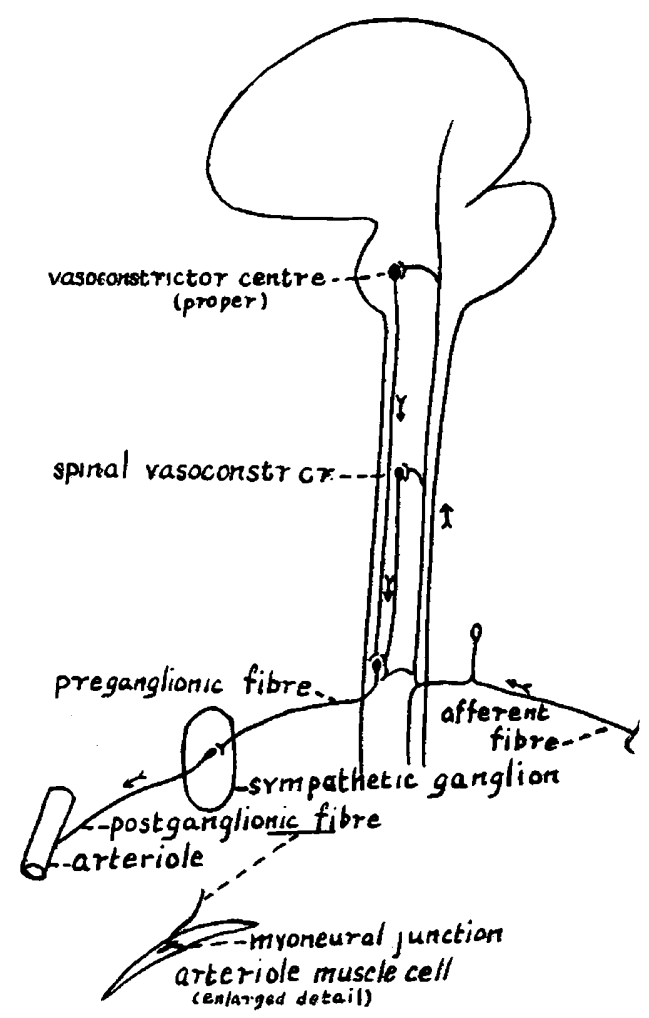

Fig. 1.-Diagrammatic scheme of the vasomotor apparatus.

The accompanying sketch (Fig. 1) illustrates the conception of the vasomotor apparatus on which our studies have been based. Although it involves nothing that is new it is included for what it may be worth in adding clearness. Suppose that a given impulse, for instance, one due to a pin prick on the leg, is destined to be delivered to the arteriole walls and cause a rise of blood pressure. The impulse travels over an afferent fiber to the spinal cord. In the cord it may go either upward to the vasoconstrictor center in the medulla or may go directly to a

1. Hoskins and Wheelon: Am. Jour. Physiol., 1914, xxxiv, 81, 172, 263; ibid, xxxv, 119; Wheelon: Ibid., 1914, xxxv, 283; Hoskins: Ibid., 1915, xxxvi, 423; Hoskins and Rowley: Hbid., 1915, xxxvii, 471. 
subsidiary center in the cord. From here it is relayed to an outgoing preganglionic fiber in one of the white rami communicantes of the sympathetic system. In one of the sympathetic ganglia it passes over to one or more postganglionic fibers, through which it finally reaches the arteriole wall. Between the terminal of this fiber and the smooth muscle cell proper on which the impulse acts is interposed a myoneural junction. This consists essentially of a "receptive substance" which bears to the muscle cell somewhat the same relation as does the percussion cap to the gunpowder of a loaded cartridge. Of the various constrictor centers that in the medulla is supposed to be of predominant importance. This is indicated by the fact that cutting the spinal cord just below the medulla usually causes a greater fall of blood pressure than does the further destruction of the cord itself.

This description takes account only of impulses which stimulate the vasoconstrictor mechanisms and cause a rise of pressure. By way of completeness it should be mentioned that other impulses cause a dilatation of the arterioles and a fall of pressure. The details in regard to the vasodilator mechanism are not at all well understood and for the purposes of this paper need not further be considered.

From the fact that curare changes the reflex irritability of the vasomotor system without a corresponding change of blood pressure level, Porter has recently concluded that there are both a vasoreflex center and a vasotonic center. ${ }^{2}$

The effects of hemorrhage on vasomotor irritability have been studied twice in recent years by American investigators. Porter ${ }^{3}$ in 1907 and he, with Marks,, in 1908 published two papers on the subject. They were concerned mostly, however, in determining the extent to which blood pressure would have to be reduced before sensory nerve stimulation would cease to be effective in changing blood pressure. It was noted that as the general blood pressure fell a given stimulus produced a smaller absolute change of level than under normal conditions. Porter maintains, however, that the absolute change of level is not a proper criterion of vasomotor irritability. He illustrates the matter by an analogy:

An unfaithful trustee robs two women. One of these has $\$ 40,000$, the other $\$ 20,000$. From each he takes $\$ 10,000$. The absolute loss is the same, but while one woman can still live on her income the other must work or beg. It is necessary, then, in measuring vasomotor reflexes to take into account the level of the blood pressure at the beginning of stimulation, and this is done by expressing the change in blood pressure as a percentage of this level.

Judged by this principle the irritability of the vasomotor center is increased by hemorrhage, since the percentile rise of pressure on

2. Porter: Am. Jour. Physiol., 1915, xxvi, 418.

3. Porter: Am. Jour. Physiol., 1907, xx, 399.

4. Porter and Marks: Am. Jour. Physiol, 1908, xxi, 460. 
stimulation is greater. Porter's analogy, however, is imperfect so far as hemorrhage is concerned. It assumes that the change in conditions during the experiment involves only the center. As a matter of fact, however, the conditions throughout the whole vasomotor mechanism are changed. We shall revert to this point in a later paragraph. Sollmann and Pilcher ${ }^{5}$ on experimental grounds deny the validity of Porter's reasoning. They found in experiments involving pressure changes due to aortic compression that as a matter of fact the percentile rise varies inversely to the level of pressure. According to this finding Porter's and Marks' experiments would indicate a loss of vasomotor irritability during even the earlier stages of hemorrhage. Pilcher and Sollmann in a later study ${ }^{6}$ have obtained results which are in direct contradiction to those just referred to. They report that any degree of hemorrhage lessens the pressor response to sciatic stimulation. This is true even when the general pressure level remains unaltered. In such a case there can be no question of "percentile change."

In view of the conflicting tenor of these late reports the matter seemed to us in need of further investigation, particularly as we were familiar with a somewhat different technic than that previously used. An obvious defect in the studies cited is that attention was fixed on the vasoconstrictor center without adequate regard to conditions in other parts of the apparatus.

Modern pharmacology, however, has afforded means of studying separately the condition of different parts of the vasomotor mechanism. This is done by injecting appropriate drugs which selectively stimulate these parts. The condition of the peripheral tissues can be tested by the use of epinephrin which stimulates the myoneural receptive substance, and, according to the best authorities, this only. If there is any question as between the myoneural junction and the muscle cell proper, pituitary extract, which stimulates the cell only, can be used. The irritability of the motor cells in the vasoconstrictor centers and the outlying ganglia can be determined by nicotin, which, in proper dosage (0.2 mg.), selectively stimulates these. ${ }^{8}$ Dosage in this connection, however, is of great importance. If large quantities are used a generalized stimulation of many structures occurs, followed shortly by a paralysis of the sympathetic ganglion cells. In this event

5. Sollmann and Pilcher: Am. Jour. Physiol., 1913, xxxi, 211.

6. Pilcher and Sollmann: Am. Jour. Physiol., 1914, xxxv, 65.

7. See, for instance, Pilcher and Sollmann: Jour. Pharm. and Exper. Therap., 1915 , vi, 339.

8. Langley and Dickinson: Jour. Physiol., 1890, xi, 297. Pilcher and Sollmann: Jour. Pharm. and Exper. Therap., 1915, vi, 369. 
vasomotor impulses are of course completely blocked. Both nicotin and epinephrin, and, to some extent, pituitary extract also, have the advantage that, in the dosages used, they can be repeated several times without significant loss of effectiveness. It is feasible, therefore, to study the changes of irritability of the vasomotor mechanism throughout the course of an experiment.

Using these methods the studies herein reported were carried out. While considerable individual variability was encountered, the essential concordance of the series as a whole permits a brief description of the results. The experiments were all made on unconscious subjects, dogs, either anesthetized with ether or decerebrated. Blood pressure from a femoral artery was recorded by means of an ordinary mercury manometer and float. The use of a Hall reservoir canula added materially to simplicity of technic. This is a glass canula of the ordinary arterial type in which is blown a 15 c.c. bulb (Fig. 2). The canula filled with 10 per cent. solution of sodium citrate can be connected by a rubber tube full of the same solution directly to the manometer. One charging of the bulb ordinarily suffices for two hours recording. There is no necessity, therefore, for the cumbersome "positive pres-

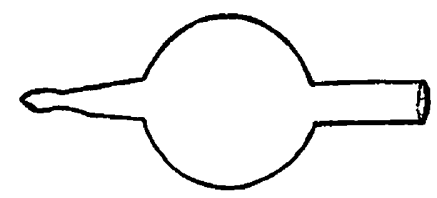

Fig. 2.-The Hall reservoir cannula, one-half size.

sure" and "wash out" arrangements commonly used in such experiments. We have used the method only with dogs. Whether it would be satisfactory with smaller animals we have not ascertained. The rate at which the drugs are injected is an essential factor in quantitative experiments. In order to keep this constant, a flushing system was employed. A large bore cannula was fastened in a femoral vein. It was connected by rubber tubing to a reservoir of 0.8 per cent. sodium chlorid solution at an elevation of two feet. The tube was clamped near the end of the cannula. The drug to be given was injected by means of a hypodermic syringe into the tube immediately above the clamp. This was at once released and the drugs instantaneously sent into the blood stream. This technic reduces the margin of experimental error to neglible proportions. Better results are secured in the study of vasomotor reflexes by cutting the vagi and thereby eliminating secondary cardio-inhibitory reflexes. Nearly all our dogs were therefore vagotomized. 


\section{THE EFFECTS OF HEMORRHAGE}

It was noted that after moderate hemorrhage the reaction to a given dose of nicotin was augmented, often to a striking degree. But the lower blood pressure that also resulted materially changed the mechanical conditions under which the vascular apparatus was working. The individual muscle cells had to contract with lower initial tension, from a shorter initial length and against a decreased load. The results were often correspondingly hard to interpret, although in certain instances the reaction was so greatly augmented as to be unequivocal. More satisfactory results were secured when, after the hemorrhage, enough isotonic saline solution was infused to restore the original pressure level and the reactions immediately determined.

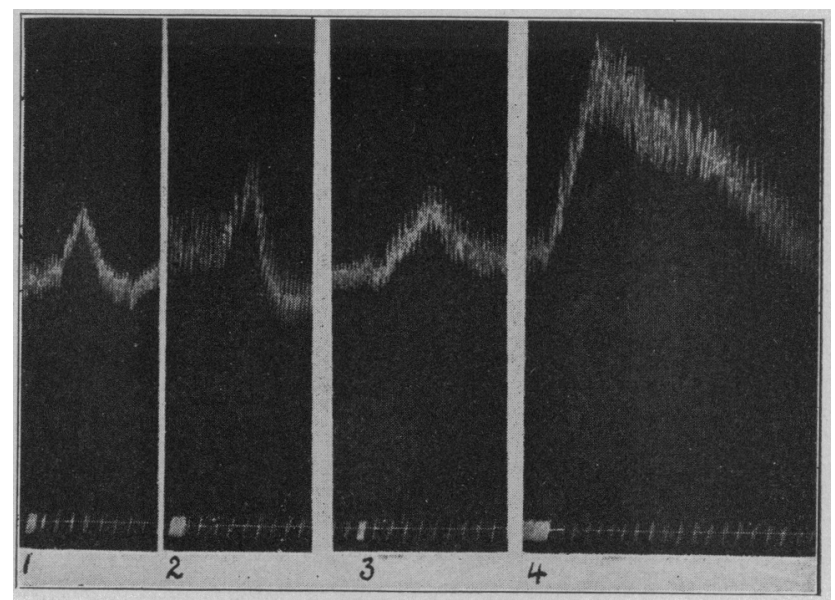

Fig. 3.-Effect of hemorrhage on vasomotor irritability. Blood pressure from femoral artery. Vagi cut. Graphs 1 and 2, reactions to 1 c.c. epinephrin (adrenalin), 1:100,000 before and after hemorrhage. Graphs 3 and 4 reactions to 1 c.c. nicotin $1: 4,000$ before and after hemorrhage ( 2 and 4 followed hemorrhage six to eight minutes). Hemorrhage 185 c.c. compensated with warm saline solution, 125 c.c. Dog weight, 11 kilos. Base line $=0-$ pressure, signals and time (five seconds). Reduced to one-half.

The fluid was of course injected at body temperature. Figure 3 illustrates the outcome of such an experiment. Graphs 1 and 2 show the reactions to 1 c.c. of epinephrin (adrenalin) $(1: 100,000)$ before and after hemorrhage. They show that little change had occurred in the condition of the peripheral parts of the apparatus. Graphs 3 and 4 show the reactions to nicotin $(1: 4,000)$ also before and after the hemorrhage. The marked augmentation of the reaction shows that the irritability of the vasomotor nerve cells had been increased. Whether the change occurred in the vasoconstrictor center, however, or in the cells of the outlying ganglia can not from such an experiment be deter- 
mined. It is probable that with the dosage used the rise of blood pressure is due largely to stimulation of the vasoconstrictor center proper, but no definite proof is available. No feasible way occurred to us to determine directly in these particular experiments which of the two sites was involved. It was supposed, however, that the essential feature of the experiments was an interference with the circulation in the medulla, leading to partial anemia of the center. Accordingly the experiments were extended as indicated in the next section.

THE EFFECT OF OCCLUDING THE CAROTID ARTERIES

The medulla gets most of its blood supply from two sources, the carotid and the vertebral arteries. While clamping the carotids leaves the medullary centers enough circulation to permit their functioning,

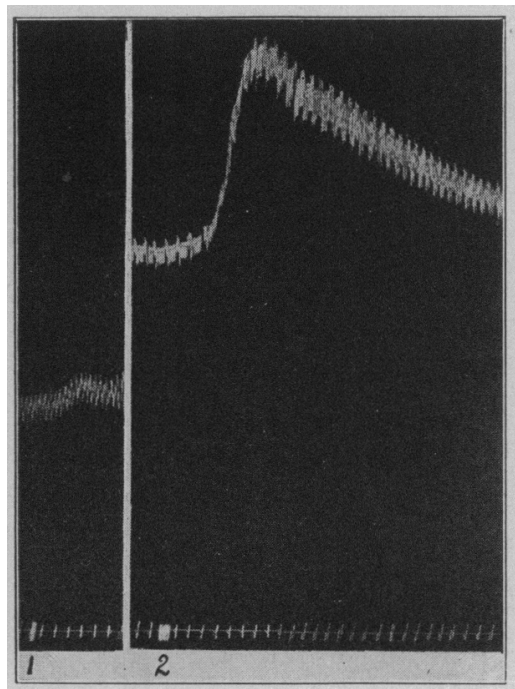

Fig. 4.-Effect of occluding carotid arteries on vasomotor irritability. Blood pressure from femoral artery. Vagi cut. Graph 1, reaction to nicotin, 1 c.c. $1: 4,000$ before occlusion. Graph 2, reaction to same dose five minutes after occlusion. Base line $=0$ - pressure, signals and time (five seconds). Reduce to one-half.

it was thought that a sufficient degree of anemia might be caused to produce an appreciable effect. In nearly every instance the procedure actually did cause a marked change in the reaction to nicotin. Figure 4 illustrates one of the more marked cases. Graph 1 shows the reaction of 1 c.c. of nicotin $(1: 4,000)$. Both carotids were then clamped. The pressure level rose to the height shown in Graph 2. Five minutes after the occlusion the same dose of nicotin now caused the much greater rise shown in Graph 2. The reactions to epinephrin were prac- 
tically the same in both cases. The essential difference, therefore, in the conditions before and after occlusion is an augmented irritability of the vasoconstrictor center. The augmentation in reaction is all the more striking because the occlusion must have partially protected the medulla from the inflow of nicotin. In the few instances in which little augmentation occurred the vertebral arteries probably were adequate to keep up a full blood supply. In one such case pressure on one vertebral artery in addition brought out the augmentation phenomenon. The concordance of both the hemorrhage and occlusion experiments indicates that the augmented irritability was in the vasoconstrictor center proper and not in the outlying ganglia. A characteristic feature of both the hemorrhage and the occlusion experiments was an increase in the length of time the pressor reaction continued.

The occlusion experiments are in harmony with clinical observations. Elevation of blood pressure is one of the accepted signs of increased intracranial pressure following contusions of the brain. But the clinical observations do not, in themselves, show whether the elevated pressure is due to increased irritability of the vasoconstrictor center or to increased stimulation of the center, perhaps by asphyxial products, or to both. The two conditions are allied but by no means identical. That irritability can be greatly augmented without concomitant stimulation is well known to any one familiar with the pharmacology of strychnin. Parenthetically it might be added that "pressure on the medulla" can not, as such, account for the raised blood pressure following brain edema. Slowly developing pressure does not stimulate but depresses.

The underlying feature in all these cases is probably an increase in the hydrogen ions in the cells concerned. Anything which interferes with the circulation of a tissue tends to cause an accumulation of acid products, such as carbon dioxid and lactic acid. This, for example, is probably the essential element in the so-called "hyperemia" treatment (Bier's), which amounts to partial asphyxia and an attendant increase of local tissue activity. A somewhat similar phenomenon is seen in the familiar process of "warming up" of athletes, or, in its laboratory guise, the "stair-case" phenomenon. In each case the efficiency of the mechanism is improved by use. The conventional explanation is that the activity liberates acid products which augment the irritability of the tissues. ${ }^{9}$

The augmented irritability of the vasoconstrictor center described in this paper would seem to have some significance as an adaptive arrangement. Several factors are recognized as aiding in compensation after hemorrhage. Such are the characteristic rapid

9. Lee: Am. Jour. Physiol., 1907, xx, p. 170. 
heart beat, the passive contraction of the vascular channels due to the inherent elasticity of the vessel walls, and the withdrawal of fluids from the tissue to restore the volume of the circulatory medium. Augmented vasoconstrictor irritability leading to higher vasomotor tonus would cooperate with these other factors in maintaining pressure and keeping up circulation in the brain at the expense of outlying tissues. Similarly when the circulation in the brain is impeded by intracranial pressure the augmented vasomotor tonus by raising systemic pressure aids in keeping the vital centers supplied. This significance of hypertension is of course well recognized.

In this paper only moderate degrees of hemorrhage have been considered ( 10 to 20 c.c. per kilo of body weight). As is well known, more extensive loss of blood or serious arrest of its circulation from any cause quickly induces a marked depression of the whole central nervous system. In this depression the vasoconstrictor center of course shares.

\section{SUMMARY AND CONCLUSIONS}

1. Certain drugs selectively stimulate different components of the vasomotor apparatus. It is possible by their use to detect changes of irritability in these components.

2. Hemorrhage causes a marked augmentation of the reaction to nicotin but slight or no augmentation of the reaction to epinephrin.

3. Occluding the carotids in most instances leads to similar results.

4. The augmented reactions are probably due, therefore, to increased irritability of the vasoconstrictor center.

5. The phenomenon is probably an adaptive arrangement tending to preserve normal circulatory conditions. 